Arq. Bras. Med. Vet. Zootec., v.66, n.2, p.602-608, 2014

\title{
Características da carcaça e dos componentes não carcaça de cordeiros oriundos de parto simples ou duplo
}

\author{
[Carcass and non-carcass traits of single and twin lambs] \\ T.P. Wommer ${ }^{1}$, C.C. Pires $^{2}$, D.B. Galvani ${ }^{3}$, M. Hastenpflug ${ }^{1}$, A.M.S. Bolzan ${ }^{2}$, \\ J.F. Lopes ${ }^{2}$, L. Griebler ${ }^{2}$, S. Carvalho ${ }^{2}$ \\ ${ }^{1}$ Instituto Federal de Educação - Ciência e Tecnologia de Mato Grosso do Sul - IFMS - Ponta Porã, MS \\ ${ }^{2}$ Universidade Federal de Santa Maria - UFSM - Santa Maria, RS \\ ${ }^{3}$ Embrapa Caprinos e Ovinos - Sobral, CE
}

\begin{abstract}
RESUMO
Um experimento foi conduzido com o objetivo de avaliar as características da carcaça e dos componentes não carcaça de cordeiros nascidos de parto simples ou duplo. Dezesseis cordeiros mestiços Texel $\times$ Ile de France, machos não castrados, foram desmamados aos 56 dias de idade e abatidos com aproximadamente $28 \mathrm{~kg}$ de peso corporal. Imediatamente após o abate, cada componente corporal foi pesado separadamente, sendo as carcaças avaliadas após 24 horas de refrigeração a $2^{\circ} \mathrm{C}$. Os cordeiros oriundos de parto simples apresentaram superioridade no peso e nos rendimentos da carcaça, além de maior área de olho de lombo. Por outro lado, proporcionalmente ao peso de corpo vazio, o rúmen é maior em cordeiros nascidos de parto duplo, o que resulta incremento da proporção de conteúdo gastrintestinal ao abate. Os resultados deste estudo indicam que, quando abatidos a um mesmo peso, cordeiros oriundos de parto simples possuem carcaças de melhor qualidade, em comparação a cordeiros nascidos de parto gemelar.
\end{abstract}

Palavras-chave: confinamento, Longissimus, ovinos, rendimento de carcaça, rúmen, vísceras

\begin{abstract}
An experiment was conducted to evaluate the carcass and non-carcass traits of single and twin lambs. Sixteen Texel $\times$ Ile de France crossbred, non-castrated male lambs were weaned at 56 days of age and slaughtered at $28 \mathrm{~kg}$ of body weight. Immediately after the slaughter each body component was individually weighed, while carcasses were evaluated after a $24 \mathrm{~h}$ period of refrigeration at $2{ }^{\circ} \mathrm{C}$. Single lambs presented higher values of carcass weight and carcass dressing percentage. Moreover, the Longissimus muscle area was also greater for single lambs. On the other hand, as a proportion of empty body weight, rumen was greater for twin lambs, increasing their gastrointestinal content at slaughter. The results of this study indicated that at a same slaughter weight single lambs have better quality carcasses in comparison with those from twin lambs.
\end{abstract}

Keywords: feedlot, Longissimus, sheep, carcass dressing percentage, rumen, viscera

\section{INTRODUÇÃO}

O nascimento de dois ou mais cordeiros por parto é uma característica marcante na espécie ovina. Determinada por fatores genéticos (Rosati et al., 2002) e ambientais (Lassoued et al., 2004), esta característica tem sido intensamente estudada, de forma a aumentar a oferta de

Recebido em 13 de junho de 2012

Aceito em 7 de novembro de 2013

E-mail: tatiana.wommer@ifms.edu.br cordeiros para abate e diluir os custos de produção.

Cordeiros nascidos de partos gemelares, todavia, geralmente apresentam peso ao nascer inferior ao dos cordeiros provenientes de partos simples (Yilmaz et al., 2007), o que pode elevar sua taxa de mortalidade (Mukasa-Mugerwa et al., 2000). Além disso, o desempenho pós-natal de 
cordeiros nascidos de parto múltiplo pode ser prejudicado, em parte devido à inabilidade dos cordeiros pequenos para ingerir o colostro nas primeiras horas de vida, mas, sobretudo, em virtude da menor ingestão individual de leite (Snowder e Glimp, 1991). Como consequência, estes animais podem permanecer mais tempo nos sistemas de terminação, consumindo maior quantidade de alimento. De qualquer forma, o maior número de cordeiros produzidos a cada estação reprodutiva é considerado vantajoso por grande parte dos produtores, e a utilização de tecnologias nutricionais que visam elevar a prolificidade do rebanho (Robinson et al., 2006) é uma prática bastante comum, uma vez que esta é a característica de maior impacto econômico quando o objetivo da criação é a produção de carne (Souza e Moraes, 2010).

São escassos, todavia, estudos acerca das características da carcaça e dos componentes não carcaça de animais oriundos de parto múltiplo. Nesse sentido, como a composição corporal e a da carcaça são diretamente afetadas pela taxa de crescimento (Oddy e Sainz, 2002), o tipo de parto pode resultar em carcaças com características distintas. Esta informação, no atual cenário de crescimento da produção de carne ovina, torna-se indispensável para padronização do produto ofertado, com vistas ao atendimento das exigências do mercado consumidor.

Este estudo foi conduzido com o objetivo de avaliar as características da carcaça e dos componentes do peso corporal de cordeiros provenientes de parto simples ou duplo, terminados em confinamento.

\section{MATERIAL E MÉTODOS}

O trabalho foi realizado no Laboratório de Ovinocultura do Departamento de Zootecnia da Universidade Federal de Santa Maria, Santa Maria - RS. Foram utilizados 16 cordeiros, machos não castrados, oriundos do cruzamento alternado contínuo estabilizado entre as raças Texel e Ile de France, sendo oito cordeiros nascidos de parto simples e oito nascidos de parto duplo. Os animais foram desmamados aos 56 dias de idade, com peso médio de $16,77 \pm 4,87 \mathrm{~kg}$, vermifugados e mantidos em baias individuais $\left(2 \mathrm{~m}^{2}\right)$, providas de comedouros e bebedouros. Periodicamente, foi realizado o monitoramento dos animais quanto às infecções por endoparasitos pelo método Famacha (Van Wyk e Bath, 2002).

A dieta experimental foi composta por $55,0 \%$ de silagem de sorgo (Sorghum bicolor (L.) Moench), 15,9\% de grão de milho triturado (Zea mays L.) e $29,1 \%$ de farelo de soja (Glycine max L.), sendo calculada de acordo com o NRC (Nutrient..., 2007). A suplementação mineral foi fornecida separadamente. Os cordeiros foram alimentados ad libitum, em horários preestabelecidos, às oito horas e às 16h30min, até que atingissem o peso de abate estabelecido, que foi de $28 \mathrm{~kg}$, segundo recomendações de Galvani et al. (2008a). A quantidade fornecida foi ajustada diariamente, visando manter as sobras em, aproximadamente, $10 \%$ do total fornecido. As sobras de alimentos foram retiradas dos cochos diariamente, antes do arraçoamento matutino, evitando a ocorrência de fermentações indesejáveis. Amostras da dieta experimental foram coletadas semanalmente para posterior realização de análises químicas (Tab. 1), conforme metodologias previamente descritas por Galvani et al. (2008b).

Tabela 1. Formulação e composição química da dieta experimental

\begin{tabular}{lccc} 
& Silagem & Concentrado & Ração total \\
\hline Matéria seca (\%) & 34,1 & 78,2 & 51,7 \\
Matéria orgânica (\% na MS) & 91,4 & 98,8 & 94,3 \\
Proteína bruta (\% na MS) & 6,2 & 33,0 & 16,9 \\
Fibra em detergente neutro (\% na MS) & 64,5 & 19,7 & 46,6 \\
Fibra em detergente ácido (\% na MS) & 45,9 & 6,8 & 30,3 \\
Extrato etéreo (\% na MS) & 3,04 & 4,71 & 3,70 \\
Carboidratos totais (\% na MS) & 82,1 & 61,1 & 73,7 \\
Carboidratos não fibrosos (\% na MS) & 17,7 & 41,3 & 27,1 \\
\hline
\end{tabular}

MS = matéria seca. 
Por ocasião do abate, os animais foram submetidos a jejum prévio de sólidos por 14 horas, sendo, em seguida, pesados para obtenção do peso ao abate (PV), insensibilizados e sacrificados mediante secção das veias jugulares e artérias carótidas. O sangue foi, então, recolhido em recipiente apropriado e pesado. Após esfola e evisceração, cada componente corporal foi pesado separadamente. $\mathrm{O}$ conteúdo gastrintestinal, a bile e a urina foram descartados para obtenção do peso corporal vazio (PCV): $\mathrm{PCV}=\mathrm{PV}-$ (conteúdo gastrintestinal + bile + urina). $\mathrm{O}$ peso de cada componente não carcaça foi expresso em relação ao PCV dos animais.

As carcaças foram pesadas para obtenção do peso de carcaça quente (PCQ) e, mediante a relação entre o PV e o PCQ, obteve-se o rendimento de carcaça quente (RCQ, \% = $(\mathrm{PCQ} / \mathrm{PV}) \times 100)$. Em seguida, as carcaças foram mantidas em câmara frigorífica a $2^{\circ} \mathrm{C}$ por 24 horas, penduradas pelos tendões de Aquiles em ganchos apropriados para manutenção das articulações tarsometatarsianas, distanciadas em, aproximadamente, $15 \mathrm{~cm}$ uma da outra. Transcorrido o período de resfriamento, as carcaças foram novamente pesadas para obtenção do peso de carcaça fria (PCF), do rendimento de carcaça fria $(\mathrm{RCF}, \%=(\mathrm{PCF} / \mathrm{PV})$ $\times 100)$ e do índice de quebra ao resfriamento $(\mathrm{IQR}, \%=((\mathrm{PCQ}-\mathrm{PCF}) / \mathrm{PCQ}) \times 100)$.

A avaliação das características quantitativas e qualitativas das carcaças dos animais foi conduzida de acordo com as metodologias estabelecidas por Osório et al. (1998). A largura de garupa foi mensurada com auxílio de compasso e, em seguida, as carcaças foram seccionadas longitudinalmente ao meio, obtendose duas meias carcaças. Na metade esquerda, mensuraram-se o comprimento de carcaça, o comprimento de perna, a largura de perna, a profundidade de perna, o perímetro de perna e a profundidade de peito. A compacidade da carcaça foi determinada pela razão entre o PCF e o comprimento da carcaça, sendo expressa em $\mathrm{kg} / \mathrm{cm}$. A área de olho de lombo (AOL) foi obtida pela exposição do músculo Longissimus dorsi após um corte transversal na carcaça entre a $12^{\mathrm{a}}$ e $13^{\mathrm{a}}$ costelas, traçando-se o contorno do músculo em papel vegetal. A área foi, então, mensurada com auxílio do programa computacional AutoCAD (AutoCAD release 14.0). A meia carcaça direita foi separada em quatro cortes (perna, paleta, costela e pescoço), e, após cada corte ser pesado individualmente, a perna foi dissecada em gordura, músculos $\mathrm{e}$ ossos.

Os dados foram analisados em delineamento inteiramente ao acaso, com oito repetições, usando-se o procedimento GLM do SAS (Statistical Analysis System). O modelo adotado incluiu o efeito fixo do tipo de parto, além do erro aleatório residual. As médias foram obtidas por meio da opção LSMEANS e comparadas pelo teste $\mathrm{F}$ da análise de variância. Todas as análises foram realizadas ao nível de $5 \%$ de significância.

\section{RESULTADOS E DISCUSSÃO}

Os cordeiros nascidos de parto simples apresentaram maiores pesos e rendimentos de carcaça quente e fria $(\mathrm{P}<0,05 ;$ Tab. 2). O rendimento de carcaça é diretamente afetado pelo desenvolvimento do trato gastrintestinal (Galvani et al., 2008a; Galvani et al., 2010) que, por sua vez, responde ao consumo de alimento sólido. Nesse sentido, o menor consumo de leite por cordeiros nascidos de parto duplo (Snowder e Glimp, 1991) e o consequente maior estímulo ao consumo de alimentos sólidos no período prédesmame (Davis et al., 1998) resultam em maior desenvolvimento ruminal e conferem maior capacidade de ingestão aos animais. Assim, quando abatidos em um mesmo peso, é possível que animais oriundos de parto múltiplo apresentem menores pesos de carcaça em comparação aos animais de parto simples, em razão de maior proporção de conteúdo gastrintestinal (CGI), em relação ao peso vivo ao abate. De fato, essa hipótese é confirmada no presente estudo, uma vez que as proporções de CGI foram, respectivamente, 19,1 e 22,2\% $(\mathrm{P}<0,05)$ para os animais nascidos de partos simples e duplo. AgFACT (1997) também reportou que cordeiros nascidos de parto duplo apresentam carcaças menores que aqueles de parto simples, quando abatidos a um mesmo peso. Todavia, naquele estudo, a magnitude da diferença observada $(0,2$ a $0,3 \mathrm{~kg})$ foi menor quando comparada aos resultados do presente trabalho, o que possivelmente está relacionado à utilização de animais com menor aptidão para produção de carne e, portanto, com menor potencial de crescimento. 
Tabela 2. Características da carcaça de cordeiros, de acordo com o tipo de parto

\begin{tabular}{|c|c|c|c|c|}
\hline & \multicolumn{2}{|c|}{ Tipo de parto } & \multirow[b]{2}{*}{$\mathrm{EPM}^{1}$} & \multirow[b]{2}{*}{$P>\mathrm{F}^{2}$} \\
\hline & Simples & Duplo & & \\
\hline Peso de abate $(\mathrm{kg})$ & 28,2 & 27,8 & 0,095 & 0,09 \\
\hline Peso de carcaça quente $(\mathrm{kg})$ & 13,2 & 11,9 & 0,143 & 0,01 \\
\hline Peso de carcaça fria $(\mathrm{kg})$ & 12,6 & 11,4 & 0,164 & 0,01 \\
\hline Rendimento de carcaça quente (\%) & 46,6 & 43,3 & 0,540 & 0,01 \\
\hline Rendimento de carcaça fria (\%) & 44,4 & 41,4 & 0,642 & 0,04 \\
\hline Índice de quebra ao resfriamento (\%) & 4,65 & 4,24 & 0,504 & 0,07 \\
\hline Comprimento da carcaça $(\mathrm{cm})$ & 52,5 & 51,6 & 0,379 & 0,24 \\
\hline Comprimento da perna $(\mathrm{cm})$ & 35,3 & 34,8 & 0,301 & 0,36 \\
\hline Largura da garupa $(\mathrm{cm})$ & 24,4 & 23,3 & 0,336 & 0,14 \\
\hline Largura da perna $(\mathrm{cm})$ & 10,6 & 9,12 & 0,349 & 0,05 \\
\hline Profundidade da perna $(\mathrm{cm})$ & 14,1 & 13,8 & 0,369 & 0,71 \\
\hline Profundidade do peito $(\mathrm{cm})$ & 22,7 & 21,9 & 0,217 & 0,10 \\
\hline Perímetro da perna $(\mathrm{cm})$ & 37,7 & 36,2 & 0,299 & 0,03 \\
\hline Compacidade da carcaça $(\mathrm{kg} / \mathrm{cm})$ & 0,24 & 0,22 & 2,550 & 0,01 \\
\hline Área de olho de lombo $\left(\mathrm{cm}^{2}\right)$ & 15,6 & 12,9 & 0,300 & 0,04 \\
\hline
\end{tabular}

${ }^{\mathrm{T}}$ Erro-padrão residual da média.

${ }^{2}$ Probabilidade do erro do Tipo I.

O índice de quebra ao resfriamento médio foi de $4,45 \%$ e não foi afetado pelo tipo de parto $(\mathrm{P}>0,05)$. Da mesma forma, não houve diferenças entre os tratamentos $(\mathrm{P}>0,05)$ para os comprimentos da carcaça e da perna, bem como para a largura de garupa e as profundidades da perna e peito. Estes resultados não estão de acordo com o de Silva e Pires (2000), que observaram correlação significativa do peso da carcaça com suas medidas; todavia, estes autores trabalharam com peso de carcaça variando entre 1,7 e $13,7 \mathrm{~kg}$. Por outro lado, os cordeiros nascidos de parto simples apresentaram maiores largura e perímetro de perna $(\mathrm{P}<0,05)$, além de possuírem carcaças com maior índice de compacidade $(\mathrm{P}<0,05)$ do que os animais oriundos de parto duplo. Entre as medidas da carcaça, estas últimas estão altamente correlacionadas com sua musculosidade (Albertí et al., 2005). Assim, tais diferenças podem estar associadas ao menor desenvolvimento das fibras musculares de cordeiros gêmeos recém-nascidos (Mccoard et al., 1997), que ocorre como consequência do menor crescimento destes durante a gestação. Este fator pode explicar, ainda, a maior área de olho de lombo apresentada pelos animais nascidos de parto simples $(15,6 \mathrm{vs}$. 12,9) e sugere que o desenvolvimento muscular fetal afeta diretamente o desenvolvimento pósparto.
Os maiores pesos de carcaça observados para os animais oriundos de parto simples resultaram em maiores pesos de paleta e perna, em relação aos animais nascidos de parto duplo $(\mathrm{P}<0,05$; Tab. 3). Neste último corte houve, ainda, incremento das quantidades de músculo e gordura $(\mathrm{P}<0,05)$ em favor dos cordeiros de parto simples. Quando expressos em valores percentuais, contudo, não houve diferenças entre os tipos de parto no tocante ao tamanho dos cortes, tampouco quanto à composição física da perna $(\mathrm{P}>0,05)$. Galvani et al. (2008a), em trabalho com animais do mesmo grupo racial dos utilizados no presente estudo abatidos entre 15 e $45 \mathrm{~kg}$ de peso corporal, observaram que apenas a proporção de paleta foi alterada à medida que o peso de abate foi elevado; embora, em pequena magnitude $(21,8 \mathrm{e}$ $20,3 \%$, para animais abatidos aos 15 e $45 \mathrm{~kg}$, respectivamente) Além disso, estes autores não observaram variação na proporção de músculo da perna, cujo valor médio foi de $70,7 \%$, bastante próximo ao do presente trabalho. Já com relação às proporções de ossos e gordura, Galvani et al. (2008a) relataram importante variação destas à medida que os animais cresceram, porém trabalharam com variações médias de $3 \mathrm{~kg}$ no peso das carcaças. 
Tabela 3. Cortes da carcaça e composição física da perna de cordeiros oriundos de parto simples ou duplo

\begin{tabular}{lcccc} 
& \multicolumn{2}{c}{ Tipo de parto } & & \\
\cline { 2 - 3 } & Simples & Duplo & & \\
\cline { 2 - 3 } Composição da carcaça & & & & \\
Pescoço (kg) & 0,51 & 0,46 & 0,02 & 0,27 \\
Costela (kg) & 2,23 & 2,12 & 0,08 & 0,16 \\
Paleta (kg) & 1,24 & 1,11 & 0,02 & 0,02 \\
Perna (kg) & 2,18 & 2,00 & 0,03 & 0,02 \\
Pescoço (\%) & 8,00 & 7,99 & 0,16 & 0,98 \\
Costela (\%) & 36,2 & 37,1 & 0,45 & 0,96 \\
Paleta (\%) & 20,2 & 19,6 & 0,15 & 0,69 \\
Perna (\%) & 35,6 & 35,2 & 0,30 & 0,48 \\
Composição física da perna & & & & \\
Gordura (kg) & 0,24 & 0,19 & 0,01 & 0,02 \\
Osso (kg) & 0,43 & 0,40 & 0,01 & 0,38 \\
Músculo (kg) & 1,50 & 1,41 & 0,02 & 0,03 \\
Gordura (\%) & 10,9 & 9,40 & 0,51 & 0,36 \\
Osso (\%) & 20,0 & 20,1 & 0,57 & 0,85 \\
Músculo (\%) & 68,6 & 69,7 & 0,67 & 0,54 \\
\hline
\end{tabular}

${ }^{1}$ Erro-padrão residual da média.

${ }^{2}$ Probabilidade do erro do Tipo I.

Não houve efeito do tipo de parto sobre a proporção (\% do PCV) da quase totalidade dos componentes não carcaça avaliados, com exceção do rúmen e dos pulmões+traqueia $(\mathrm{P}<0,05)$, que foram maiores para os animais nascidos de parto duplo, e da proporção de gordura renal, que foi superior para os cordeiros de parto simples $(\mathrm{P}<0,05 ;$ Tab. 4). A maior proporção de rúmen para os animais oriundos de parto múltiplo, como discutido anteriormente, possivelmente está relacionada ao maior estímulo ao consumo de alimentos sólidos no período pré-parto, e respalda os resultados observados para os pesos e rendimentos de carcaça. Além disso, Pires et al. (2006) sugeriram que cordeiros gêmeos apresentaram maior proporção de rúmen + retículo + omaso + abomaso devido ao maior tempo de permanência desses animais no confinamento, o que resulta em maior consumo de alimento sólido. Esta observação é corroborada pelos resultados do presente estudo, uma vez que os cordeiros nascidos de parto duplo alcançaram o peso de abate, em média, com 40 dias de atraso $(\mathrm{P}<0,05)$ em relação àqueles de parto simples (dados não apresentados). De fato, se considerados conjuntamente, rúmen + retículo + omaso + abomaso representam 3,47 e $3,89 \%$ do PCV, e são maiores nos cordeiros gêmeos $(\mathrm{P}<0,05)$. Por sua vez, a maior proporção de pulmão+traqueia encontrada para os cordeiros gêmeos está de acordo com a observação de Galvani et al. (2010) de que este órgão diminui de tamanho, em relação ao $\mathrm{PCV}$, à medida que os animais crescem. Estes autores, ao avaliarem o crescimento dos diferentes componentes corporais, relataram proporção média de pulmão+traqueia de $1,65 \%$ do $\mathrm{PCV}$, para cordeiros de parto simples abatidos aos $30 \mathrm{~kg}$ de $\mathrm{PV}$, semelhante ao valor obtido no presente estudo.

As diferenças encontradas neste estudo quanto à proporção de gordura renal podem estar igualmente relacionadas à diferença de PCV dos animais, uma vez que esse depósito de gordura tende a aumentar com incremento do peso dos animais (Galvani et al., 2010). Além disso, uma provável maior taxa de ganho dos animais nascidos de parto simples, sobretudo no período pré-desmama, pode ter contribuído para estes resultados, visto que existe estreita relação entre a taxa de ganho e a deposição de gordura corporal (Oddy e Sainz, 2002). As proporções dos demais componentes corporais avaliados são 
semelhantes àquelas relatadas por Pires et al. (2006) e Galvani et al. (2010).

Em síntese, os resultados observados no presente estudo indicam superioridade das carcaças dos animais nascidos de parto simples, o que está relacionado, sobretudo, ao maior peso de carcaça desses animais. Em razão disso, é possível que um incremento do peso de abate dos cordeiros gêmeos resulte em carcaças semelhantes àquelas dos cordeiros de parto simples. Com base na proporção de conteúdo gastrintestinal observada neste estudo, estima-se que tal incremento deveria ser da ordem de $4 \%$. Esta afirmativa, contudo, deve ser comprovada experimentalmente.

\section{CONCLUSÃO}

Quando abatidos a um mesmo peso, cordeiros oriundos de parto simples possuem carcaças de melhor qualidade, em comparação a cordeiros nascidos de parto gemelar.

\section{COMITÊ DE ÉTICA E BIOSSEGURANÇA}

Aprovado pelo Comitê de Ética Animal da Universidade Federal de Santa Maria - UFSM Número do Parecer: 007/2011.

\section{REFERÊNCIAS}

AgFACT. Dressing percentages in lambs. Nova Zelândia: Ruakura Research Centre, 1997. (Report n.227).

ALBERTÍ, P.; RIPOLL, G.; GOYACHE, F. et al. Carcass characterisation of seven spanish beef breeds slaughtered at two commercial weights. Meat Sci., v.71, p.514-521, 2005.

DAVIS, J.J; SAHLU, T.; PUCHALA, R. et al. Performance of Angora goat kids fed acidified milk replacer at two levels of intake. Small Rumin. Res., v.28, p.249-255, 1998.

GALVANI, D.B.; PIRES, C.C.; WOMMER,T.P. et al. Carcass traits of feedlot crossbred lambs slaughtered at different live weights. Cienc. Rural, v.38, p.1711-1717, 2008a.

GALVANI, D.B.; PIRES, C.C.; KOZLOSKI G.V., et al. Energy requirements of Texel crossbred lambs. J. Anim. Sci., v.86, p.34803490, 2008b.
GALVANI, D.B.; PIRES, C.C.; WOMMER, T.P. et al. Postweaning growth of the noncarcass components of Texel crossbred lambs. Rev. Bras. Zootec., v.39, p.1558-1564, 2010.

LASSOUED, N.; REKIK, M.; MAHOUACHI, M. et al. The effect of nutrition prior to and during mating on ovulation rate, reproductive wastage, and lambing rate in three sheep breeds. Small Rumin. Res., v.52, p.117-125, 2004.

MCCOARD, S.A.; PETERSON, S.W.; MCNABB, W.C. et al. Maternal constraint influences muscle fibre development in fetal lambs. Reprod. Fertil. Dev., v.9, p.675-682, 1997.

MUKASA-MUGERWA, E.; LAHLOU-KASSI, A.; ANINDO, D. et al. Between and within breed variation in lamb survival and the risk factors associated with major causes of mortality in indigenous Horro and Menz sheep in Ethiopia. Small Rumin. Res., v.37, p.1-12, 2000.

NUTRIENT requirements of small ruminants: Sheep, goats, cervids, and new world camelids. Washington, D.C.: National Academy Press, 2007. 384p.

ODDY, V.H.; SAINZ, D.R. Nutrition for sheepmeat production. In: FREER, M.; DOVE, H. (Ed.). Sheep Nutrition. Wallingford, UK: CABI Publishing, 2002. p.237-262.

OSÓRIO, J.C.S.; OSÓRIO, M.T.M.; JARDIM, P.O. et al. Métodos para avaliação da produção de carne ovina: 'in vivo', na carcaça e na carne. Pelotas: UFPEL, 1998. 98p.

PIRES, C.C.; CARNEIRO, R.M.; MULLER, L. et al. Avaliação da carcaça e componentes do peso vivo de cordeiros de parto simples desmamados, parto simples não desmamados e de parto duplo desmamados. Rev. Bras. Agrocienc., v.12, p.93-97, 2006.

ROBINSON, J.J.; ASHWORTH, C.J.; ROOKE, J.A. et al. Nutrition and fertility in ruminant livestock. Anim. Feed Sci. Technol., v.126, p.259-276, 2006.

ROSATI, A.; MOUSA, E; VAN VLECK, L.D. et al. Genetic parameters of reproductive traits in sheep. Small Rumin. Res., v.43, p.65-74, 2002.

SILVA, L.F.; PIRES, C.C. Avaliações quantitativas e predição das proporções de osso, músculo e gordura da carcaça em ovinos. Rev. Bras. Zootec., v.29, p.1253-1260, 2000. 
SNOWDER, G.D.; GLIMP, H.A. Influence of breed, number of suckling lambs, and stage of lactation on ewe milk production and lamb growth under range conditions. J. Anim. Sci., v.69, p.923-930, 1991.

SOUZA, C.J.H.; MORAES, J.C.F. Como utilizar a genética Booroola. Bagé: Embrapa Pecuária Sul, 2010. 4p. (Comunicado técnico, 73).
VAN WYK, J.A.; BATH, G.F. The FAMACHA system for managing haemonchosis in sheep and goats by clinically identifying individual animals for treatment. Vet. Res., v.33, p.509-529, 2002.

YILMAZ, O.; DENK, H.; BAYRAM, D. et al. Effects of lambing season, sex and birth type on growth performance in Norduz lambs. Small Rumin. Res., v.68, p.336-339, 2007. 